\title{
SHORT REPORT \\ Overwhelming post-splenectomy sepsis in patients with asplenia and hyposplenia: a retrospective cohort study
}

\author{
J. CHONG ${ }^{1,2}$, P. JONES ${ }^{1}$, D. SPELMAN ${ }^{1}$, K. LEDER ${ }^{2}$ AND A. C. CHENG ${ }^{1,2 *}$ \\ ${ }^{1}$ Department of Infectious Diseases, Alfred Health, Melbourne, VIC, Australia \\ ${ }^{2}$ Department of Epidemiology and Preventive Medicine, Monash University, Melbourne, VIC, Australia
}

Received 2 June 2016; Final revision 21 September 2016; Accepted 27 September 2016;

first published online 25 October 2016

\section{SUMMARY}

Overwhelming post-splenectomy infection (OPSI) is a serious complication of asplenia and is associated with encapsulated organisms, most commonly Streptococcus pneumoniae, but also Haemophilus influenzae and Neisseria meningitidis. We aimed to estimate the risk of infection in this patient group. We reviewed data collected by the Victorian Spleen Registry in Australia. On registration, all patients are asked about significant infections requiring admission to hospital for intravenous antibiotics; those requiring admission to ICU were defined as OPSI. In the 3274 asplenic patients registered 492 patients reported at least one episode of infection. There were 47 episodes of OPSI requiring intensive care (incidence rate $1 \cdot 11 / 1000$ patient-years). The risk of OPSI was highest in older patients, and there were no statistically significant differences in incidence by reason for splenectomy except for a higher rate in patients with medical hyposplenia. This study reinforces that post-splenectomy infection is a clinically significant but uncommon complication, and that fulminant infection requiring intensive care is a minority of all infections.

Key words: Bacterial infection, cohort study, registry, splenectomy, sepsis.

Overwhelming post-splenectomy infection (OPSI) is a serious complication of asplenia, and associated with significant morbidity and mortality [1]. Although variously defined, patients with asplenia are at particular risk of infection with encapsulated organisms, most commonly Streptococcus pneumoniae, but also Haemophilus influenzae and Neisseria meningitidis. A number of measures are recommended to reduce the risk of OPSI, including patient education, vaccination against encapsulated organisms, prophylactic antibiotics and the provision of an infection action plan including the ready availability of emergency antibiotics

\footnotetext{
* Author for correspondence: Professor A. C. Cheng, Department of Epidemiology and Preventive Medicine, Monash University, Commercial Road, Melbourne, VIC 3004, Australia.

(Email: allen.cheng@monash.edu)
}

$[2,3]$. However, many studies have found that adherence to preventative measures is generally poor [4].

The Victorian Spleen Registry (VSR) has operated since 2003, and enrols patients who are asplenic or hyposplenic in the state of Victoria, Australia. The VSR aims to increase the awareness of postsplenectomy infection in asplenic patients by education of patients and their healthcare providers, and to disseminate up-to-date recommendations to prevent infection [5]. Patients are routinely asked about their experience with infection requiring hospitalization at the time of registration. All cases of infection requiring ICU admission were confirmed by review of the hospital discharge summary. On registration, each patient's GP is contacted to confirm key details including details of splenectomy. Additionally, the registry maintain contact with patients via periodic 
newsletters and surveys, as well as ad hoc clinical queries from patients and providers. The registry is sent hospital discharge summaries for non-ICU admissions, but this is not systematically performed. In this study, we aimed to describe the rate of overwhelming post-splenectomy sepsis in patients with asplenia and hyposplenia.

For this retrospective cohort study, we reviewed data collected on infections at the time of registration to the VSR. We defined post-splenectomy infection as an episode of suspected or confirmed infection treated as a hospital inpatient with intravenous antibiotics. We defined a subset of these infections to be OPSI that required admission to intensive care, which were categorized as being caused due to encapsulated organisms (S. pneumoniae, $H$. influenza, $N$. meningitidis), due to other organisms, and those which were culture negative. We excluded patients who were registered within 1 month of splenectomy, as we believed that infections in this period would be more likely to reflect post-operative complications. Consistent with accepted terminology, we refer to infection associated with medical hyposplenia as OPSI, with the date of 'splenectomy' being the date of diagnosis of hyposplenia. Similarly, we excluded patients who were registered within 1 month of the diagnosis of hyposplenism to reduce the possibility that they were registered because of an episode of infection.

We calculated the cumulative proportion of patients who had experienced post-splenectomy infection or OPSI prior to registration with the VSR. We excluded patients who did not reside in Victoria, and patients who had infection before or within 1 month of splenectomy. We maintain data on severe infection following registration based on passive surveillance via patients and clinician networks, but the completeness of ascertainment is unclear. The cumulative incidence of infection was defined as the number of patients experiencing at least one episode of infection divided by the patients at risk prior to registration or after registration. The relative rate of infection was calculated with confidence intervals assuming the Poisson distribution. We constructed a multivariate model for OPSI using a Poisson regression with age group and reason for splenectomy as dependent variables, accounting for time at risk. Statistical analyses were performed using Stata v. 13 for Windows (StataCorp., USA).

Ethical permission to review these data was granted by the Human Research Ethics Committee at Alfred Health and Monash University.
At March 2014, there were 3274 VSR registrants, of whom $221(6.7 \%)$ had died. Of these patients, the median age at splenectomy was 38 years (interquartile range 22-58 years) and the mean time since splenectomy was 11 years. There were $1767(54 \%)$ registered within 6 months of splenectomy.

At least one episode of asplenia- or hyposplenia-associated infection prior to registration was reported in 492 patients. This proportion rose from $9 \%$ in the 1670 patients registered within the last 3 years, to $22 \%$ in the 1577 patients registered more than 3 years since splenectomy (Table 1).

There were 47 episodes of OPSI recorded in the 3274 patients prior to registration. Of these, 17 infections were due to encapsulated bacteria (pneumococcus $n=16, H$. influenzae $n=1), 15$ due to other bacteria (Aspergillus spp. $n=1$; coagulase-negative staphylococcus $n=1$, Escherichia coli $n=2$, group B streptococcus $n=1, H$. influenzae (non-sterile site) $n$ $=2$, Klebsiella spp. $n=2$, Serratia spp. $n=2$; Staphylococcus aureus $n=2$, Streptococcus bovis $n=$ 1) and 15 presented with culture-negative severe infection. The risk of severe infection was highest for the first 3 years after splenectomy and lower thereafter.

In a multivariate model taking into account the varying time at risk, which was greater for younger patients, the incidence rate of severe infection was higher in patients who had splenectomy at an older age (age at splenectomy $>65$ years [relative rate (RR) 5.03, 95\% (CI) 1.88-13.47], <20 years (RR $0 \cdot 30,95 \%$ CI $0 \cdot 14-0 \cdot 67)$ vs. 20-64 years). The relative rate of severe infection (compared to those with traumatic splenectomy) was similar for non-malignant haematological conditions (RR 0.71, 95\% CI $0 \cdot 30$ 1.66), malignant haematological conditions (RR $0 \cdot 30,95 \%$ CI $0 \cdot 04-2 \cdot 24$ ), other cancer (RR 0.59, $95 \%$ CI 0.08-4.56), incidental splenectomy (RR 1.26 95\% CI 0.40-3.96) but higher for those with medical hyposplenia (RR $10 \cdot 12,95 \%$ CI $4 \cdot 15-24 \cdot 64$ ).

The 47 OPSI cases recorded in the 38681 patientyears prior to registration compared to four identified OPSI cases in the 12370 patient-years following registration, giving a relative rate of OPSI following registration of 0.29 (95\% CI $0 \cdot 076-0 \cdot 80, P=0.003)$

The risk of OPSI in patients with asplenia is difficult to determine from published studies, due to differences in patient populations and definitions of OPSI used. In a review of 28 studies including 6942 patients with a median follow-up of $6 \cdot 9$ years, Bisharat and colleagues estimated that the cumulative incidence of invasive infection was around 3\% [6]. However, different studies 
Table 1. Rates of OPSI prior to registration on the Victorian Spleen Registry

\begin{tabular}{|c|c|c|c|c|c|c|c|c|}
\hline & \multirow[b]{2}{*}{$\begin{array}{l}\text { No. of } \\
\text { patients }\end{array}$} & \multirow{2}{*}{$\begin{array}{l}\text { Period of } \\
\text { observation } \\
\text { (person-years } \\
\text { of follow-up) }\end{array}$} & \multicolumn{3}{|c|}{$\begin{array}{l}\text { Infection requiring } \\
\text { hospitalization }\end{array}$} & \multicolumn{3}{|c|}{$\begin{array}{l}\text { Infection requiring intensive } \\
\text { care }\end{array}$} \\
\hline & & & No. & Risk* & $\begin{array}{l}\text { Relative risk } \dagger \\
(95 \% \mathrm{CI})\end{array}$ & No. & Rate* & $\begin{array}{l}\text { Relative rate } \dagger \\
(95 \% \mathrm{CI})\end{array}$ \\
\hline All patients & 3274 & $38681 \cdot 0$ & 492 & $0 \cdot 15$ & & 43 & $1 \cdot 11$ & \\
\hline \multicolumn{9}{|c|}{ Age at splenectomy, years } \\
\hline$<20$ & 652 & $17355 \cdot 4$ & 124 & $0 \cdot 19$ & 1 & 8 & $0 \cdot 46$ & 1 \\
\hline $20-65$ & 2055 & $20598 \cdot 5$ & 306 & $0 \cdot 15$ & $0.78(0 \cdot 64-0.94)$ & 30 & $1 \cdot 46$ & $3 \cdot 16(1 \cdot 42-7 \cdot 97)$ \\
\hline$>65$ & 564 & $633 \cdot 1$ & 62 & $0 \cdot 11$ & $0.58(0.44-0.77)$ & 5 & $7 \cdot 90$ & $17 \cdot 13(4 \cdot 41-59 \cdot 4)$ \\
\hline \multicolumn{9}{|l|}{ Indication } \\
\hline Trauma & 1027 & $17429 \cdot 4$ & 170 & $0 \cdot 17$ & 1 & 16 & $0 \cdot 92$ & 1 \\
\hline $\begin{array}{l}\text { Non-malignant } \\
\text { haematological }\end{array}$ & 754 & $11639 \cdot 9$ & 115 & $0 \cdot 15$ & $0 \cdot 92(0 \cdot 74-1 \cdot 14)$ & 8 & $0 \cdot 69$ & $0 \cdot 75(0 \cdot 28-1 \cdot 85)$ \\
\hline $\begin{array}{l}\text { Malignant } \\
\text { haematological }\end{array}$ & 245 & $2684 \cdot 9$ & 34 & $0 \cdot 14$ & $0 \cdot 84(0 \cdot 60-1 \cdot 18)$ & 1 & $0 \cdot 37$ & $0 \cdot 41(0 \cdot 01-2 \cdot 61)$ \\
\hline Cancer & 290 & $1020 \cdot 9$ & 36 & $0 \cdot 12$ & $0 \cdot 75(0 \cdot 54-1 \cdot 05)$ & 1 & $0 \cdot 98$ & $1 \cdot 07(0 \cdot 02-6 \cdot 87)$ \\
\hline Incidental & 325 & $1822 \cdot 9$ & 50 & $0 \cdot 15$ & $0.93(0 \cdot 70-1 \cdot 24)$ & 4 & $2 \cdot 19$ & $2 \cdot 39(0 \cdot 58-7 \cdot 41)$ \\
\hline Hyposplenia & 110 & $710 \cdot 0$ & 21 & $0 \cdot 19$ & $1 \cdot 15(0 \cdot 64-1 \cdot 47)$ & 7 & $9 \cdot 86$ & $10 \cdot 74(3 \cdot 74-27 \cdot 6)$ \\
\hline Other reasons & 523 & $3373 \cdot 0$ & 66 & $0 \cdot 13$ & $0.76(0.59-0.99)$ & 6 & $1 \cdot 78$ & 1.94 \\
\hline \multicolumn{9}{|c|}{$\begin{array}{l}\text { Time since splenectomy, } \\
\text { years }\end{array}$} \\
\hline$<3$ & 1670 & $334 \cdot 8$ & 146 & $0 \cdot 09$ & 1 & 13 & $38 \cdot 83$ & 1 \\
\hline $3-10$ & 304 & $1909 \cdot 8$ & 68 & $0 \cdot 22$ & $2 \cdot 56(0 \cdot 97-3 \cdot 32)$ & 7 & $3 \cdot 67$ & $0.09(0.03-0.25)$ \\
\hline $10-30$ & 774 & $15308 \cdot 0$ & 167 & $0 \cdot 22$ & $2 \cdot 47(2 \cdot 01-3 \cdot 03)$ & 10 & $0 \cdot 65$ & $0.02(0.006-0.042)$ \\
\hline$>30$ & 525 & $21128 \cdot 6$ & 111 & $0 \cdot 21$ & $2.42(1.92-3.03)$ & 13 & $0 \cdot 62$ & $0.02(0.006-0.037)$ \\
\hline
\end{tabular}

OPSI, Overwhelming post-splenectomy infection, CI, confidence interval.

* We were unable to determine if patients had more than one non-severe infection, so infection requiring hospitalization was reported as a cumulative risk, but patients with severe infection was reported as a rate per 1000 patient-years.

$\dagger$ Univariate analysis.

have used various definitions of post-splenectomy infection, including any hospitalization with an infectious diagnosis [7, 8], invasive pneumococcal disease [9], pneumonia, meningitis or septicaemia [10], 'overwhelming' infection $[10,11]$ and infection requiring ICU admission [12]. We found that our patient group had a similar cumulative incidence of infection compared to studies where a broader definition was used (patient self-report of infection requiring hospitalization for intravenous antibiotics); for example, a Victorian study using hospital administrative data found that $26 \%$ of patients with surgical splenectomies had a subsequent readmission with an infectious diagnosis [8]. The incidence of infection when defined by criteria that reflect severe sepsis (e.g. 'fulminant' infection) appears to be much lower $[10,11]$.

We were surprised to find that there were few severe infections following splenectomy due to haematological malignancies and a particularly high rate in patients with medical hyposplenia. Previous studies have found higher rates of infection were associated with non-malignant haematological conditions (particularly sickle cell disease and thalassaemia), but similar rates of infection in patients with trauma [6]. Previous studies have suggested that younger age at splenectomy is associated with a higher risk of OPSI. Although we had no splenectomized patients aged $<2$ years, we found an increasing OPSI rate with age once the time at risk was accounted for, similar to the findings in a Scottish population-based study [11]. Although we excluded episodes of infection in hyposplenic patients within 1 month of diagnosis to reduce the possibility that they may have been registered due to an episode of infection, the estimated incidence of severe infection should be interpreted with caution in this group.

While we were not able to establish the timing of infection relative to splenectomy, the varying durations of time between splenectomy and registration do provide us with an indirect measure of risk over time. We found that the proportion with reported postsplenectomy infections increased over the first 3 years then were stable thereafter. This is consistent with previous data suggesting an increased risk of infection early after splenectomy [11]. 
There are several limitations to our study. Because our method of ascertainment of infection relies on patient self-report at the time of registration, we may have underestimated the true incidence of severe infection, as patients who die may not come to our attention. Alternatively, it is also possible that patients with OPSI might be more likely to seek health advice and register with the VSR. The wording on the survey question did not enumerate the episodes of infection, so we were unable to determine if patients had more than one (non-severe) infection. Our results therefore indicate a cumulative risk rather a true incidence. Although we actively sought discharge summaries in patients who had severe infections, clinical details were not consistently available for non-severe infections. Data regarding episodes of post-splenectomy sepsis following registration should be viewed with caution as under-ascertainment is possible.

This study reinforces the view that postsplenectomy infection is a clinically significant but uncommon complication, and that fulminant infection requiring intensive care represents a minority of all infections. Several studies elsewhere have shown that adherence to measures to prevent infection in this group are poor [13-16]. The VSR improves adherence to these measures by providing patient and healthcare provider education, updates and disseminates recommendations on vaccine and other preventive measures and facilitates research into asplenia [5]. Further evaluation regarding the effectiveness of the registry via linkage with notifiable disease databases and additional prospective studies would be informative.

\section{ACKNOWLEDGEMENTS}

We thank Stacey Rowe and Nicola Stephens at the Victorian Department of Health for their support. The VSR is funded by Alfred Health and the Victorian Department of Health and Human Services. A.C. is supported by a National Health and Medical Council Career Development Fellowship. The funders had no role in the conduct of this study.

\section{DECLARATION OF INTEREST}

None.

\section{REFERENCES}

1. Di Sabatino A, Carsetti R, Corazza GR. Post-splenectomy and hyposplenic states. Lancet 2011; 378: 86-97.

2. Jones $\mathbf{P}$, et al. Postsplenectomy infection - strategies for prevention in general practice. Australian Family Physician 2010; 39: 383-386.

3. Spelman D, et al. Guidelines for the prevention of sepsis in asplenic and hyposplenic patients. Internal Medicine Journal 2008; 38: 349-356.

4. El-Alfy MS, El-Sayed MH. Overwhelming postsplenectomy infection: is quality of patient knowledge enough for prevention? Hematology Journal 2004; 5: 77-80.

5. Wang $\mathbf{J}$, et al. Adherence to infection prevention measures in a statewide spleen registry. Medical Journal of Australia 2014; 200: 538-540.

6. Bisharat $\mathbf{N}$, et al. Risk of infection and death among post-splenectomy patients. Journal of Infection 2001; 43: $182-186$.

7. Thomsen RW, et al. Risk for hospital contact with infection in patients with splenectomy: a population-based cohort study. Annals of Internal Medicine 2009; 151: 546-555.

8. Dendle C, et al. Splenectomy sequelae: an analysis of infectious outcomes among adults in Victoria. Medical Journal of Australia 2012; 196: 582-586.

9. Foss Abrahamsen A, et al. Systemic pneumococcal disease after staging splenectomy for Hodgkin's disease 1969-1980 without pneumococcal vaccine protection: a follow-up study 1994. European Journal of Haematology 1997; 58: 73-77.

10. Cullingford GL, et al. Severe late postsplenectomy infection. British Journal of Surgery 1991; 78: 716-721.

11. Kyaw MH, et al. Evaluation of severe infection and survival after splenectomy. American Journal of Medicine 2006; 119: 276 e271-277.

12. Theilacker C, et al. Overwhelming postsplenectomy infection: a prospective multicenter cohort study. Clinical Infectious Disease 2016; 62: 871-878.

13. Coignard-Biehler $\mathbf{H}$, et al. Adherence to preventive measures after splenectomy in the hospital setting and in the community. Journal of Infection and Public Health 2011; 4: 187-194.

14. Meerveld-Eggink A, et al. Vaccination coverage and awareness of infectious risks in patients with an absent or dysfunctional spleen in the Netherlands. Vaccine 2008; 26: 6975-6979.

15. Waghorn DJ. Overwhelming infection in asplenic patients: current best practice preventive measures are not being followed. Journal of Clinical Pathology 2001; 54: 214-218.

16. O'Donnell J, et al. Management of patients undergoing splenectomy in an Irish teaching hospital: impact of guidelines. Irish Journal of Medical Science 2004; 173: $136-140$. 